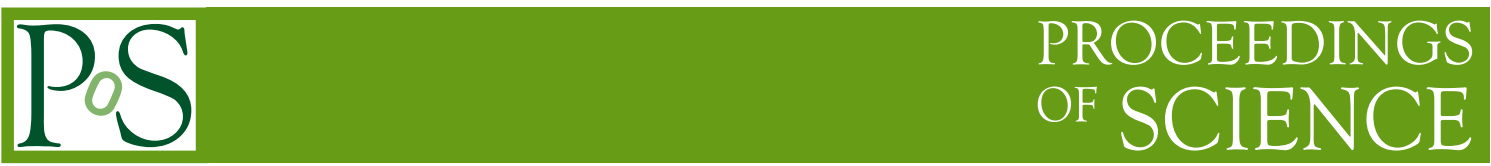

\title{
On the sources of high energy neutrinos
}

\author{
Andrea Palladino* \\ Deutsches Elektronen-Synchrotron (DESY), Platanenallee 6, Zeuthen (Germany) \\ E-mail: andrea.palladino@desy.de
}

\begin{abstract}
The discovery of a diffuse flux of high energy neutrinos has opened a new era in the field of neutrino astronomy. Up to now only one high energy neutrino has an identified astrophysical counterpart, the blazar TXS 0506+056. However the origin of the diffuse neutrino flux remains still a mystery, even if many possible explanations have been proposed in the last few years. The most natural hypothesis was that high energy neutrinos are produced by blazars, since these powerful objects dominate the $\gamma$-ray sky above $100 \mathrm{TeV}$. However the IceCube stacking limit shows that resolved blazars cannot contribute more than $20 \%$. Other natural sources are the ones rich of gas, in which the proton-proton interaction dominates. In this scenario an issue would be the over-production of $\gamma$-rays associated to neutrinos, if the neutrino spectrum were too soft. In this work we summarize the present knowledge and we discuss the role of low luminosity BL Lacs, showing that it is still possible to power the sub-PeV neutrino flux with blazars. Moreover we also discuss the role of $p p$ sources, showing that they are still into the game and they can saturate the sub-PeV neutrino emission, giving also a contribution larger than $50 \%$ in the energy range between $10 \mathrm{TeV}$ and $100 \mathrm{TeV}$.
\end{abstract}

The New Era of Multi-Messenger Astrophysics - Asterics2019

25 - 29 March, 2019

Groningen, The Netherlands

${ }^{*}$ Speaker. 


\section{The IceCube measurements}

The discovery of a diffuse flux of high energy neutrinos made by IceCube in 2012 [1] has opened a new window in the field of neutrino astronomy and astroparticle physics. Up to now only one neutrino event has been associated with a known astrophysical object, the blazar called TXS $0506+056$ [2]. A blazar is an Active Galactic Nuclei, with the emitted jet pointing to the Earth. Other neutrinos still remain without any satisfying astrophysical counterpart, therefore the origin of the diffuse neutrino flux is still an open question.

Nowadays there are different measurements of the astrophysical neutrino flux covering the energy range between roughly $1 \mathrm{TeV}$ and $10 \mathrm{PeV}$, obtained using different techniques. Before proceeding let us recall that in neutrino telescopes two kinds of events can be observed: tracks and showers. Track-like events are produced by the charged current interaction of $v_{\mu}$ (and negligibly by $\left.v_{\tau}\right)$ and they are characterized by a good angular resolution $\left(\sim 1^{\circ}\right.$ in ice, sub degree in water $)$ and a poor energy resolution. Shower-like events are produced by charged current interactions of $v_{e}$ and $v_{\tau}$ plus neutral current interaction of all neutrino flavors; opposite to throughgoing muons, they are characterized by a good energy resolution and a poor angular resolution $\left(\sim 15^{\circ}\right.$ in ice, few degrees in water). Let us summarize the dataset available at the present:

- the High Energy Starting Events (HESE) dataset includes all the contained events, i.e. the ones in which neutrinos are produced by deep inelastic scattering inside the detector. This technique is sensitive to all neutrino flavors (both tracks and showers) and the energy threshold is $60 \mathrm{TeV}$. This dataset suggest a soft power law spectrum $E^{-\alpha}$, with $\alpha=2.92_{-0.29}^{+0.33}$ after 6 years of data taking [3];

- the throughgoing muon flux is only sensitive to muon neutrinos that interact via charged current interaction (i.e. only track-like events), with the vertex of interaction usually outside the detector. These events are characterized by having an energy larger than $200 \mathrm{TeV}$ and they suggest a harder spectrum compared to HESE, with $\alpha=2.19 \pm 0.10$ after 8 years of data taking [3];

- the cascade sample contains low energy events (only showers), above $1 \mathrm{TeV}$. It suggests a spectrum with an intermediate shape compared to HESE and throughgoing muons, with a spectral index $\alpha=2.48 \pm 0.08$ after 4 years of detection [3].

A summary of these IceCube measurements is represented in the left panel of Fig.1. According to the last IceCube analyses [3], a single power law spectrum is still sufficient to describe all the measurements, although a broken power law spectrum with a hardening at multi-TeV energy improves the agreement with the combined data. Moreover no hint for a peculiar shape of the neutrino spectrum (different from the simple power law) is contained in the IceCube measurements. Is the spectrum of astrophysical neutrinos giving some suggestion on the production mechanism? We investigate this aspect in the next section.

\section{Production mechanisms}

The typical interactions in which high energy neutrinos are expected to be produced are protonproton [4] and proton-gamma interactions [5]. The first one is likely to occur in astrophysical 

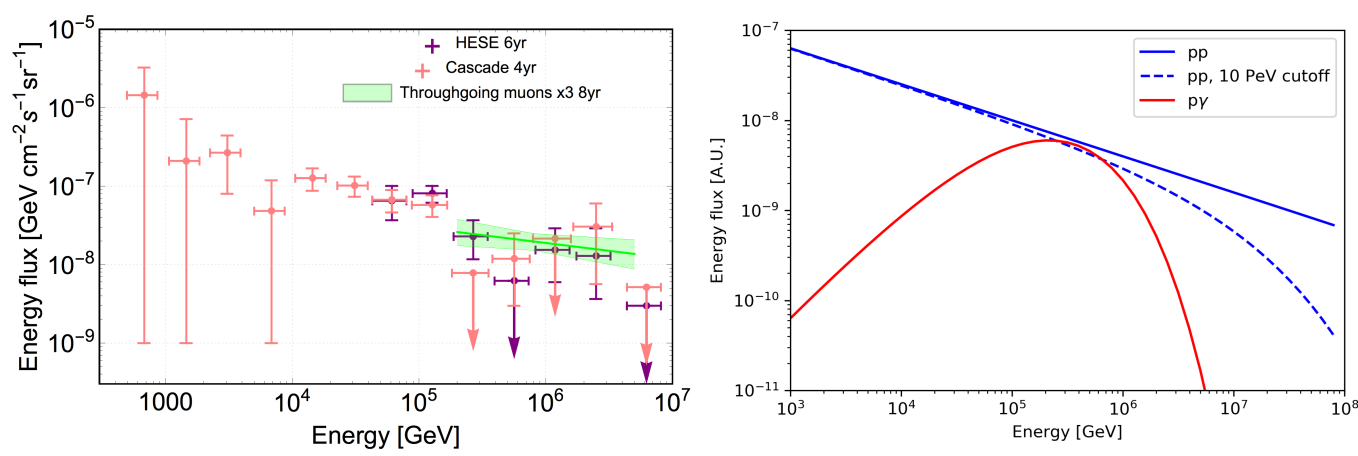

Figure 1: On the left panel: summary of the IceCube data as reported in [3], namely 6 years of High Energy Starting Events (purple points), 8 years of throughgiong muons (green band) and 4 years of Cascade events (pink points). On the right panel: examples of theoretical neutrino spectra expected from proton-proton interaction (blue curves) and one spectrum expected from proton-gamma interaction (red curve).

environments rich of gas, like Star Forming Galaxies, while the second one is likely to occur in sources rich of radiations, like Active Galactic Nuclei (AGNs). From the previous two interactions, pions are produced and from their decay high energy neutrinos come out, taking roughly $1 / 20$ of the primary particle's energy. Apart from that, the neutrino spectra from $p p$ and $p \gamma$ interactions are very different. Indeed in the first case the neutrino spectrum replicates the spectrum of the primary protons; a power law remains a power law in this case. In the $p \gamma$ interactions the neutrino spectrum does not replicate the primary spectrum; in this case the energy spectrum assumes a typical bump shape, as illustrated in the right panel of Fig.1 (red curve). In this figure we can also see some example of $p p$ neutrinos without energy cutoff (blue curve), with energy cutoff at $10 \mathrm{PeV}$ (dashed blue curve) and one example of $p \gamma$ neutrinos (red curve). Up to now the IceCube measurements does not show any indication of $p \gamma$ interaction, since they can be described by a power law spectrum extending for many order of magnitude (at least from $\mathrm{TeV}$ to $\mathrm{PeV}$ energies). However a larger exposure is required to better clarify this important aspect.

\section{AGNs vs Star Forming Galaxies}

Blazars are Active Galactic Nuclei with the emitted jet pointing to Earth. These objects dominate the $\gamma$-ray emission above $100 \mathrm{GeV}$, therefore it is natural to expect that they significantly contribute also to the high energy neutrino flux. However the lack of spatial correlations between IceCube neutrinos and known blazars constrain the contribution of visible sources at level of $20 \%$ [6]. It is important to remark that this limit only applies to resolved sources.

The efficiency of neutrino production in blazars, defined as $L_{v} / L_{\gamma}$, increases with the increasing of the $\gamma$-ray luminosity and then it becomes roughly flat for high luminosity blazars [7]. Therefore $L_{v} \propto L_{\gamma}^{2}$ for low luminosity BL Lacs and $L_{v} \propto L_{\gamma}$ for high luminosity ones. In [8] it has been shown that low luminosity BL Lacs (mainly unresolved objects) may give an important contribution to the neutrino flux at sub-PeV energy, under the assumption that these objects are rich of protons. In that model the contribution of high luminosity sources, that would violate the IceCube stacking limit, is suppressed reducing the amount of protons in high luminosity objects. In conclusions the 

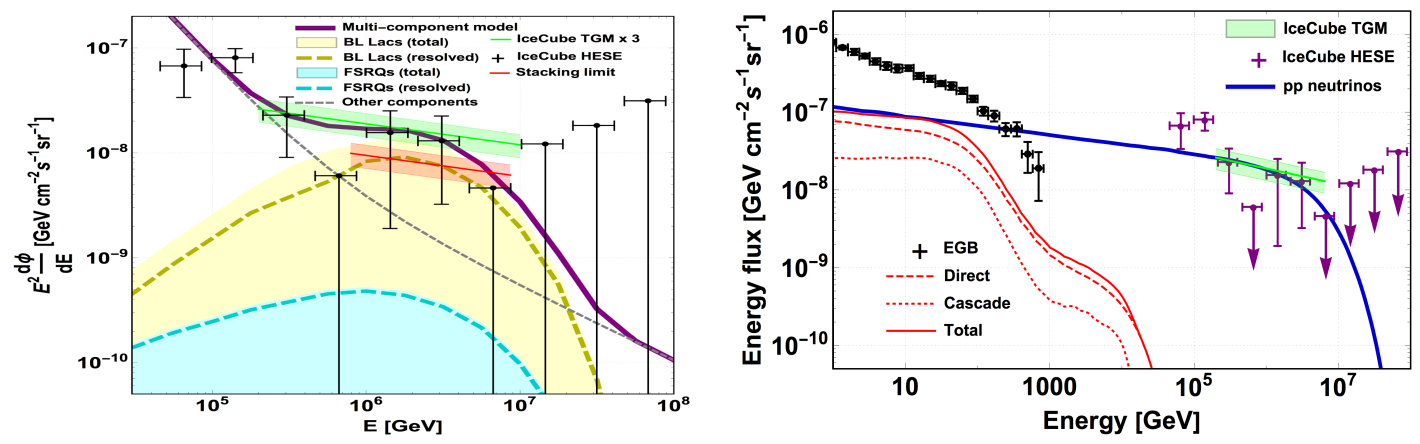

Figure 2: On the left panel: interpretation of the most energetic IceCube events in terms of blazars ( $p \gamma$ source, yellow band) [8]. On the right panel: interpretation of the most energetic IceCube data in terms of $p p$ sources (blue curve) [11]. The red solid curve shows the associated $\gamma$-ray background.

baryonic loading (defined as the ratio between the proton and $\gamma$-ray luminosity) should quickly decrease with the increasing of the $\gamma$-ray luminosity, in order to "save" blazars as neutrino emitters. Adding other components (such as residual atmospheric neutrinos, Galactic neutrinos, prompt neutrinos) it could be even possible to saturate the flux measured by IceCube (see the purple curve in the left panel of Fig.2).

Another possibility, as discussed above, is that high energy neutrinos are produced by $p p$ interaction, that it is likely to occur in Star Forming Galaxies and Starburst Galaxies. These powerful hadronic sources are natural accelerators and plausible neutrino emitters. In this case the issue is that, being the neutrino spectrum a power law, the $\gamma$-ray flux associated to high energy neutrinos would be too high for soft neutrino spectrum [9]. This would violate the non-blazar contribution to the Extragalactic Gamma-Ray Background (EGB) [10], that is $14 \% \pm 14 \%$ according to FermiLAT analysis. This problem can be avoided under the hypothesis that the true extragalactic neutrino spectrum is hard and close to $E^{-2.1}$, as suggested by throughgoing muons. Such a spectrum may saturate the high energy part of the neutrino spectrum (above $100 \mathrm{TeV}$ ), contributing to about 50\% of the energy flux between $10 \mathrm{TeV}$ and $100 \mathrm{TeV}$ [11]. This result is shown in the right panel of Fig.2 using a $E^{-2.12}$ neutrino spectrum (blue curve); also the associated $\gamma$-ray flux is shown (red curve) in comparison with the detected extragalactic $\gamma$-ray background (black points). In this scenario an additional component is required to explain part of the neutrino flux below $100 \mathrm{TeV}$. In [12] it has been discussed the possibility that the low energy contribution is provided by prompt neutrinos; also in this work a hard $p p$ neutrino spectrum is used for the extragalactic component.

\section{Conclusion}

In 2012 a diffuse flux of high energy neutrinos has been discovered by IceCube. Since then only one astrophysical counterpart has been found, namely the blazar TXS 0506+056 in 2018. The origin of the high energy neutrino flux remains still an unsolved problem.

The IceCube analysis indicates the compatibility between data and a single power law spectrum, although a broken power law spectrum with a hardening at multi-TeV energies improves the agreement with the data. No hint of proton-gamma interaction is present in the shape of the neutrino spectrum. 
Possible candidate sources are blazars (in which the $p \gamma$ interaction dominates) and Star Forming Galaxies plus Starburst Galaxies, where the $p p$ interaction dominates. In case of blazars the contribution of resolved sources is limited to $20 \%$ by the IceCube stacking analysis, reflecting the lack of lack of spatial correlations between neutrinos and known blazars. Low-luminosity BL Lacs may provide an additional contribution without violating this limit, since they are mostly unresolved objects; in order to do that, these objects are required to be rich of protons. In this scenario it is possible to almost saturate the neutrino flux above $200 \mathrm{TeV}$, while other components are required to explain the low energy flux. A similar result is achieved using neutrinos from $p p$ sources. In this case, in order to not over-produce the $\gamma$-rays associated to the neutrino production, the neutrino spectrum has to be hard, according to what is measured using throughgoing muons. Such a spectrum may saturate the flux above $200 \mathrm{TeV}$, contributing to the $50 \%$ of the energy flux between $10 \mathrm{TeV}$ and $100 \mathrm{TeV}$. An additional contribution coming from prompt neutrinos may be relevant in the $\mathrm{TeV}$ range, as recently discussed in the literature.

Acknowledgments. This project has received funding from the European Research Council (ERC) under the European Union's Horizon 2020 research and innovation programme (Grant No. 646623).

\section{References}

[1] M. G. Aartsen et al. [IceCube Collaboration], Science 342 (2013) 1242856 doi:10.1126/science.1242856 [arXiv:1311.5238 [astro-ph.HE]].

[2] M. G. Aartsen et al. Science 361 (2018) no.6398, eaat1378 doi:10.1126/science.aat1378 [arXiv:1807.08816 [astro-ph.HE]].

[3] M. G. Aartsen et al. [IceCube Collaboration], arXiv:1710.01191 [astro-ph.HE].

[4] S. R. Kelner, F. A. Aharonian and V. V. Bugayov, Phys. Rev. D 74 (2006) 034018 Erratum: [Phys. Rev. D 79 (2009) 039901] doi:10.1103/PhysRevD.74.034018, 10.1103/PhysRevD.79.039901 [astro-ph/0606058].

[5] S. R. Kelner and F. A. Aharonian, Phys. Rev. D 78 (2008) 034013 Erratum: [Phys. Rev. D 82 (2010) 099901] doi:10.1103/PhysRevD.82.099901, 10.1103/PhysRevD.78.034013 [arXiv:0803.0688 [astro-ph]].

[6] M. G. Aartsen et al. [IceCube Collaboration], Astrophys. J. 835 (2017) no.1, 45 doi:10.3847/1538-4357/835/1/45 [arXiv:1611.03874 [astro-ph.HE]].

[7] X. Rodrigues, A. Fedynitch, S. Gao, D. Boncioli and W. Winter, Astrophys. J. 854 (2018) no.1, 54 doi:10.3847/1538-4357/aaa7ee [arXiv:1711.02091 [astro-ph.HE]].

[8] A. Palladino, X. Rodrigues, S. Gao and W. Winter, Astrophys. J. 871 (2019) no.1, 41 doi:10.3847/1538-4357/aaf507 [arXiv:1806.04769 [astro-ph.HE]].

[9] K. Bechtol, M. Ahlers, M. Di Mauro, M. Ajello and J. Vandenbroucke, Astrophys. J. 836 (2017) no.1, 47 doi:10.3847/1538-4357/836/1/47 [arXiv:1511.00688 [astro-ph.HE]].

[10] M. Ackermann et al. [Fermi-LAT Collaboration], Phys. Rev. Lett. 116 (2016) no.15, 151105 doi:10.1103/PhysRevLett.116.151105 [arXiv:1511.00693 [astro-ph.CO]].

[11] A. Palladino, A. Fedynitch, R. W. Rasmussen and A. M. Taylor, arXiv:1812.04685 [astro-ph.HE].

[12] C. Mascaretti and F. Vissani, arXiv:1904.11938 [astro-ph.HE]. 